\title{
Photo-oxidative Aging Behaviors of Acrylic Coatings during UV Irradiation Investigated by Positron Annihilation Spectroscopy
}

\author{
Xipo Zhao ${ }^{a}, \mathrm{Xiallin}_{\mathrm{Cai}}{ }^{a}, \mathrm{Chang}_{\mathrm{Liu}}{ }^{a}, \mathrm{Ting}_{\mathrm{Chen}}{ }^{a}, \mathrm{Chunqing} \mathrm{He}^{a}, \mathrm{Pengfei} \mathrm{Fang}^{b}$ \\ And ShaOXian PenG ${ }^{a}$ \\ ${ }^{a}$ Hubei Provincial Key Laboratory of Green Materials for Light Industry, \\ Collaborative Innovation Center of Green Light-weight Materials and Processing, \\ School of Materials Science and Engineering, \\ Hubei University of Technology, Wuhan 430068, China \\ ${ }^{b}$ School of Physics and Technology, Wuhan University, Wuhan, 430072, China
}

\begin{abstract}
Slow positron annihilation spectroscopy, contact angle and scanning electron microscope (SEM) were employed to investigate the surface polarity and morphologies as functions of aging time in acrylic coatings during UV-a irradiation. The $S$ parameter plots of each exposed sample are lower than that of the virgin sample in the whole range of $E$. The $S$ parameter plots become lower as the exposing longer time, indicated that the irradiation generated more polar groups and increased the oxidation layer depth. Combined with surface morphology and the surface energy of acrylic coating, the variation of contact angle, and surface energy, polar component and dispersive component is related with surface polarity and morphologies.
\end{abstract}

DOI: $10.12693 /$ APhysPolA.132.497

PACS/topics: Acrylic coating, UV irradiation, positron annihilation spectroscopy

\section{Introduction}

Acrylic based coatings are widely used as long-term protective coatings in various industrial applications [13]. During the service of acrylic coatings, variation of environmental effects such as sunlight, temperature, atmosphere, etc. can lead to the deterioration of their properties [4-6], which greatly limit their applications. In many cases, photo-oxidation is one of the most principal degradation processes. Therefore, it is necessary to understand the failure mechanism of acrylic coating under the related artificial accelerated aging test conditions (e.g. expose to UV radiation at different temperatures).

The influence of UV irradiation on the degradation of acrylic coatings has been investigated in the literature [47]. All their findings mainly focused on the photodegradation mechanism, especially chemical structure changes. UV radiation at coating surface generates a strong thermal and oxidative degradation in the presence of oxygen $[6,8]$.

Positron annihilation spectroscopy (PAS) coupled with a slow positron beam has been used to study the free volume in polymeric coatings systems [8-10]. In this paper, slow positron annihilation spectroscopy was employed to investigate the polarity features as a function of aging time in acrylic coatings during UV-a irradiation. Combined with contact angle and scanning electron micro-

e-mail: psxbb@126.com, fangpf@whu.edu.cn scope (SEM), the effects of UV radiation on the surface polarity and morphology of acrylic coatings were studied.

\section{Experimental}

\subsection{Materials and samples preparation}

One-component acrylic coating and diluent were purchased from Jiangsu Sanmu Group Corporation. The acrylic coating system was prepared by mixing onecomponent acrylic coating and recommended dosage of diluent with continuous stirring for 0.5 hour $(\mathrm{h})$ at room temperature. The samples were applied on carbon steel substrates and then cured in an oven at $60^{\circ} \mathrm{C}$ for $24 \mathrm{~h}$. The thickness of the dry films was $20 \pm 2 \mu \mathrm{m}$.

\section{2 $U V$-a degradation}

The ultraviolet (UV) treatment of acrylic coatings was performed using commercial UV-A lamps (center wavelength $340 \mathrm{~nm}$ ). The UV radiation power density of $55 \mathrm{~mW} / \mathrm{cm}^{2}$ was employed. The aging temperature was regulated at $60^{\circ} \mathrm{C}$. All the samples were exposed to UV irradiation with different exposure time: 0, 24, 121, 280, 445, 666, 1360 hours, respectively.

\subsection{Characterization}

Positron annihilation radiation Doppler Broadening Spectroscopy (DBS) was employed to characterize the micro-structural changes of the UV treated acrylic coating. The slow positrons generated with a $50 \mathrm{mCi}$ of ${ }^{22} \mathrm{Na}$, was moderated by a thin layer of solid $\mathrm{Ne}$, and then electromagnetically transported to the sample. The vacuum 
in the beam chamber was kept at approximately $10^{-8} \mathrm{~Pa}$. The DBS information recorded by a high-purity Ge solidstate detector with an energy resolution of $1.3 \mathrm{keV}$ at the $511 \mathrm{keV}$ peak, were obtained as a function of positron energy from 0 to $25 \mathrm{keV}$ at room temperature. The total count of each spectrum is $2 \times 10^{6}$ with a counting rate of $1200 \mathrm{cps}$. The positron annihilation line shape $S$ parameters from DBS were calculated as the ratio of the central area $(511-0.76 \mathrm{keV}<E<511+0.76 \mathrm{keV})$ to the total area of positron annihilation peak after the background is properly subtracted. The mean implantation depth of the positron is defined by the incident energy and is calculated by the following established equation [8]:

$$
z(E)=(400 / \rho) E^{1.6},
$$

where $z$ is the mean implantation depth $[\mathrm{nm}], \rho$ is the density of the acrylic coating $\left[\mathrm{g} / \mathrm{cm}^{3}\right], E$ is the incident energy of positron $[\mathrm{keV}]$. In this study, the density of the acrylic coating is $1.07 \mathrm{~g} / \mathrm{cm}^{3}$.

The polarity of acrylic coating samples aged by the UV irradiation was quantified by a static contact angle, which was measured with a goniometer (Minilab, ILMS, France). Five single droplets of deionized water $\left(\mathrm{H}_{2} \mathrm{O}\right)$ and diiodomethane $\left(\mathrm{CH}_{2} \mathrm{I}_{2}\right)$ in a volume of about $1 \mu \mathrm{L}$ were injected on the surface of acrylic coating samples with a micro-syringe. The drops were placed at five different positions on each of the acrylic coating sample. The surface energy, polarity component and dispersive component of acrylic coatings under different photooxidative aging time were calculated with Owens-WendtRabel-Kaelble (OWRK) model.

The morphologies of cryo-fractured and impact fractured surface of the samples were examined by SEM (JSM-6390LV, JEOR Japan) at an accelerating voltage of $30 \mathrm{kV}$.

\section{Results and discussion}

\section{$3.1 S$ parameter analysis}

Plot of $S$ parameter versus positron incident energy and positron implantation depth for acrylic coatings exposed to UV-a irradiation for different exposure time are shown in Fig. 1. The $S$ parameter is used to characterize polymer coatings degradation. In polymer coatings, the $S$ parameter the relative contribution of the low momentum part of positron-electron annihilation radiation which is related with the chemical composition, structure, and local defects like free volume and cavities. Generally, more free volume and larger cavities, and polar groups in materials can provide more trapping centers of positron, leading to higher $S$ parameter.

For virgin sample, the $S$ parameter increases rapidly from the surface to the bulk as $E$ is increasing. After reaching a certain depth, the $S$ parameter remains nearly constant. The small value of $S$ parameter at the surface is due to the backscattering and back-diffusion of the positron and Ps (after the incident positron picks up an electron) from the surface and near the surface to the vacuum. All these factors reduce the fraction of

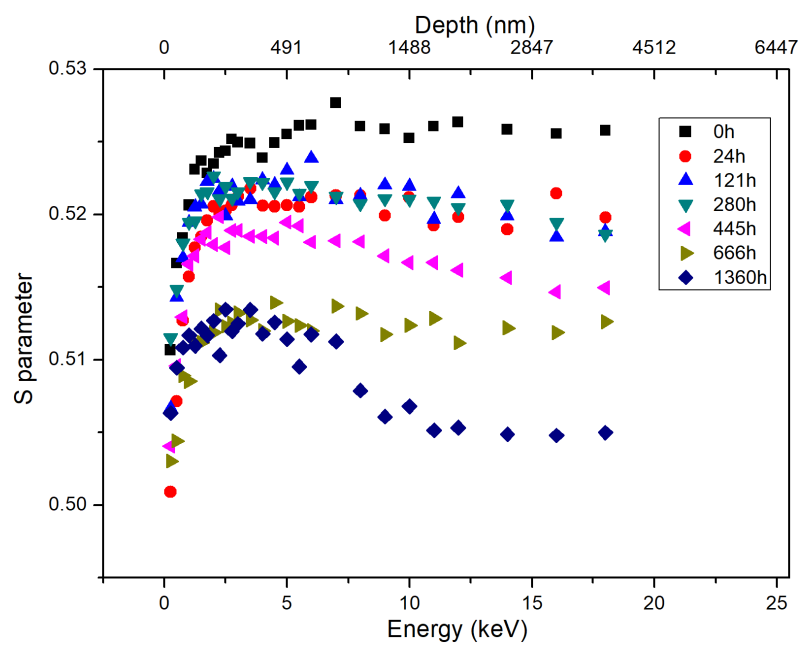

Fig. 1. $S$ parameter versus positron incident energy and positron implantation depth for acrylic coatings exposed to UV-a irradiation for different exposure time.

positron and Ps annihilated inside the materials, so that relatively lower $S$ parameter is observed near the surface. As the positron incident energy increases, $S$ parameter increases and maintains constant after reaching a certain depth (about 121nm), indicating that positrons have penetrated the coating bulk.

For UV-treated samples, the trend of the $S$ parameter is similar to that of the virgin sample. But the $S$ parameter plots of each exposed sample are lower than that of the virgin sample in the full range of $E$. In addition, as the exposure time is longer, the $S$ parameter becomes lower. For the samples irradiated 24, 121, and $280 \mathrm{~h}$, the $S$ parameter plots show negligible changes. It has been suggested that the decreases in the $S$ parameter on exposure time is a result of decreases in the defects (free volume and/or cavities) size and concentration due to irradiation $[9,10]$. At this stage, the chemical environment remains relatively stable, it is inferred that there is a more compact structure in the surface with less amount of free volumes due to UV-a irradiation.

For sample irradiated $666 \mathrm{~h}$, a rapidly decrease in $S$ parameter can be observed near the sample surface. The value of $S$ parameter increases as $E$ increases and then maintains constant. As the extension of UV irradiating time, relatively stable hydroperoxide will be produced by oxidation of the tertiary carbon. Two types of hydroperoxides are likely to be produced. The first associated with main chain oxidation will give rise to less stable hydroperoxides, and the second one is linked to side chain oxidation of the methylene adjacent to the ester function. The generation of peresters and peracids with polar groups creating much trapping centers of positrons greatly decrease the $S$ values. For sample irradiated $1360 \mathrm{~h}$, the $S$ parameter increases to the peak and then gradually decreases. This change is mainly caused by the irradiation generation much more polar groups and the oxidation depth increases gradually. 


\subsection{Static contact angle and surface polarity analysis}

Water static contact angle measurement was conducted to investigate the surface hydrophobicity variation of UV-treated and untreated coating samples. The images are shown in Fig. 2 (the images of diiodomethane static contact angle were not shown for brevity). Compared to the virgin coating sample, all the contact angles of UV irradiated samples are lower, and this could be the fact that the surface hydrophilicity of all the samples changed obviously, indicating the hydrophilicity and polarity of acrylic coatings were enhanced with increasing the UV irradiation time.

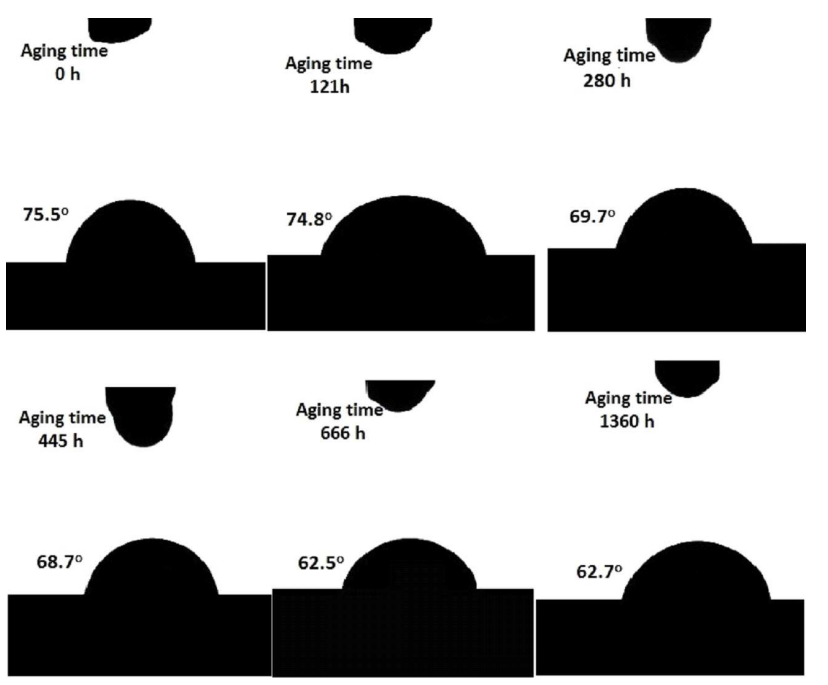

Fig. 2. Water contact angle with respect to different aging time for UV treated acrylic coatings.

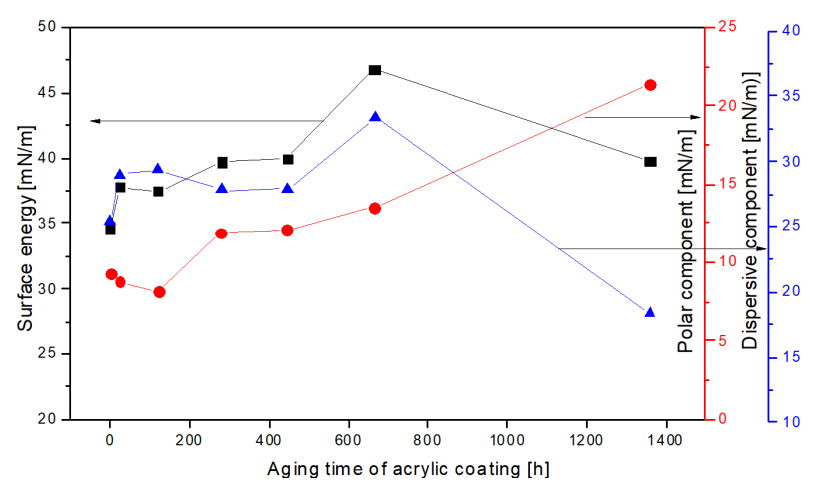

Fig. 3. Variations of surface energy, polar component and dispersive component on aging time of acrylic coatings under UV irradiation.

The surface energy of coating is used to characterize the variation of polarity. The polar component of surface energy reflects the polarity part of van der Waals force, and can be used to characterize the solid surface polarity. Dispersive component of surface energy reflects the non-polar part of van der Waals force, and can be used to characterize the morphology of the film surface. The variation of surface energy, polar component and dispersive component of acrylic coatings with different UV irradiation time are shown in Fig.3. The surface energy, polar component and dispersive component of acrylic coatings increase gradually with increasing UV irradiation time from $0 \mathrm{~h}$ to $666 \mathrm{~h}$. With increasing UV irradiation time, both surface energy and polar component of coating films increase significantly, which indicate that the hydrophilicity and polarity of acrylic coatings were improved, suggesting the peresters and peracids with polar groups for the coating surfaces were generated. This result is in agreement with the result of positron annihilation analysis. The change of polarity is mainly because the irradiation generate more polar groups and the oxidation depth increases gradually. Similarly, the increasing in dispersive component indicated the surface toughness of coatings increased. However, when the irradiation time increased to $1360 \mathrm{~h}$, the reduction of surface energy and dispersive component is unreasonable. This may be associated with the drastically changes of surface morphologies of acrylic coating.

\subsection{Morphologies observation}

To explore further the surface performance of acrylic coating, SEM was empolyed to observe the surface morphologies of the samples with different UV irradiation time, as shown in Fig. 4. Apparently, the acrylic coating
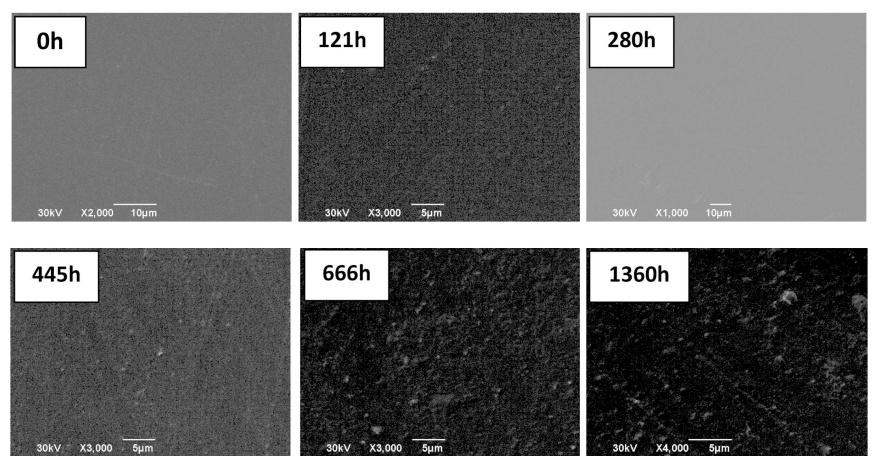

Fig. 4. SEM micrographs of acrylic coatings exposed under different UV irradiation time.

irradiated for $0,24,121$, and $280 \mathrm{~h}$ have similar morphologies with a compact and smooth morphologies. After $445 \mathrm{~h}$ and $666 \mathrm{~h}$ irradiation, the coating surfaces remained basically smooth, only the surface morphology turned rough slightly with little bumps in the coating surfaces. When the irradiating time further increased to $1360 \mathrm{~h}$, the UV irradiated sample became much rougher and more bumps appeared on the surface. It is indicated from the observation that aged coatings experienced some physical changes under the combined effects of UV irradiation and temperature, while they still remain intact where visible micro-defects were found on the surfaces. According to service life prediction theory and model for chain of events leading to macroscopic 
failure, in general, a catastrophic macroscopic failure is the result of microscopic physical changes in the coating, which in turn are usually the result of chemical changes that have occurred in the coating [11]. The morphology features under UV-irradiation for $1360 \mathrm{~h}$ can explain the reduction of surface energy and dispersive component. The changes of the surface morphologies of different UV irradiated time is consistent with the variation of surface energy, polar component and dispersive component.

Combined with surface morphology and surface energy of acrylic coating, the variations of contact angle, and surface energy, polar component and dispersive component are related with surface polarity and morphologies. After irradiated $1360 \mathrm{~h}$, the surface is much rougher, some bumps and more micro-defects can be observed, the contact angle is no longer in agreement with the Young equation.

\section{Conclusion}

The surface polarities and morphologies of acrylic coatings as function of the irradiation time were investigated by slow positron annihilation spectroscopy, contact angle and scanning electron microscope (SEM). The results indicated that the $S$ parameter plots of each exposed sample are lower than that of the virgin sample in the full range of $E$. As the exposing longer time, the $S$ parameter plots become lower, indicated that more polar groups and the oxidation depth is gradually increasing because of UV-irradiation. Combined with surface morphology and surface energy of acrylic coating, the variations of contact angle, and surface energy, polar component and dispersive component are related with surface polarity and morphologies. The contact angle is no longer in accordance with the Young equation when the irradiation time further increased to $1360 \mathrm{~h}$, and the surface is much rougher, some bumps and more micro-defects are observed.

\section{Acknowledgments}

The research was supported by the granted of National Natural Science Foundation of China (21174108, 51273060) and National Undergraduate Training Program for Innovation and Entrepreneurship (201610500004, 201710500014).

\section{References}

[1] N. Agarwal, R. Farris, J. Polym. Engin. Sci. 40, 376 (2000).

[2] S. Saha, D. Kocaefe, Y. Boluk, A. Pichette, Prog. Organ. Coat. 70, 376 (2011).

[3] F. Yang, L. Zhu, D. Han, R. Cao, W. Li, Y. Chen, Prog. Organ. Coat. 90, 455 (2016).

[4] M. Aguirre, M. Goikoetxea, L.A. Otero et al., Surf. Coat. Tech. 321, 484 (2017).

[5] C.M. Popescu, C.M. Tibirna, C. Vasile, Photochem.. Photobio. 87, 779 (2011).

[6] P. Simon M. Fratricova P. Schwarzer, H.W. Wilde Polym. Degrad. Stab. 91, 94 (2006).

[7] F.X. Perrin, M. Irigoyen, E. Aragon, J.L. Verne, Polym. Degrad. Stab. 70, 469 (2000).

[8] P.J. Schultz, K.G. Lynn, Rev. Mod. Phys. 60, 701 (1988).

[9] H. Cao, Y. He, R. Zhang, J. Yuan, T.C. Sandreczki, Y.C. Jean, J. Polym. Sci. Part B Polym. Phys. 37, 1289 (2015).

[10] X. Gu, C.A. Michaels, D. Nguyen, Y.C. Jean, J.W. Martin, T. Nguyen, Appl. Surf. Sci. 252, 5168 (2006)

[11] K.A. Wood, Prog. Organ. Coat. 43, 207 (2001). 